\title{
THE EFFECT OF THE REMOVAL OF THE NUCLEUS DENTATUS ON THE PARKINSONIAN SYNDROME
}

\author{
BY \\ SZABOLCS TÓTH \\ From the Institute of Neurosurgery, Budapest
}

Parkinsonism for long was dealt with only by
neurologists and neuropathologists, but it became
of great interest to neurosurgeons when the possi-
bility arose that operation might solve the problem.
Interventions were carried out on all those parts of
the pyramidal and extrapyramidal system which
could be considered on a pathological or experi-
mental basis to be responsible for the development of the symptoms. Since the paper by DelmasMarsalet and van Bogaert (1935) no account has been given of operations performed on the cerebellum. These authors found that after electrical coagulation of the dentate nucleus there was a slight diminution of rigidity whereas tremor became more marked. They stopped this intervention on account of complications arising in one of their patients, and as far as we know neither they nor others have since carried out operations of that kind. In the surgery of extrapyramidal diseases attention has been directed rather to interventions on the pallidothalamic system; and indeed some authors carrying out experimental research work considered that Parkinsonian tremor was of cerebellar origin (Carrea, Reissig, and Mettler, 1947, 1955). In their opinion Parkinsonian tremor is a result of a cerebellar lesion and further intervention on the cerebellum only increases it. However, according to Fulton (1949), after the isolated removal of the nucleus dentatus tremor (cerebellar intention) is of a transitory character and becomes only more marked after the removal of the nucleus globosus and emboliformis. We therefore decided to operate on the cerebellum in certain cases in order to diminish the Parkinsonian syndrome.

First, it seemed to us that hypotonia resulting from a cerebellar lesion caused by removal of the dentate nucleus might be expected to reduce rigidity but that no conclusions could be drawn from the published data concerning the question of tremor; either improvement or worsening of this symptom might follow the operation.

Secondly, it has become evident that surgical interventions on the pallido-thalamic system had not been so successful that we should not seek new operative methods to solve the problem. Again, among the successful operations on the thalamus there were some in the course of which the thalamic part of the dentato rubro-thalamic tract was disconnected. In these cases, tremor and rigidity diminished more markedly than after operations on the pallidum (Bravo and Cooper, 1959).

The possible disadvantages of causing a cerebellar syndrome had to be taken into consideration. We expected, however, that after the removal of one nucleus dentatus the disturbing side-effects would show a tendency to recover well. On the other hand, if we wanted to achieve prolonged results the efferent tracts of the cerebellum should be disconnected as extensively as possible in spite of possible prolonged side-effects. Also it was doubtful whether disconnecting the dentate nucleus in an intact extrapyramidal system would have the same effects as the operation in a pathologically defective one.

In two of our three patients we attempted a stereotactic operation on the pallido-thalamic system before interfering with the cerebellum. In one case we failed to carry out the procedure, and in the second case it did not prove very successful and the effects were not sufficiently lasting for it to seem worth while to repeat the operation.

\section{Methods}

After an incision along the midline and a suboccipital craniectomy we expose the vermis and medial parts of the hemispheres, and 3-4 $\mathrm{mm}$. lateral to the midline an incision $2 \mathrm{~cm}$. in length is made in the cortex parallel to the midline (Fig. 1). The white matter is removed together with the dentate nucleus by means of suction. We proceed sidewards, forwards, and backwards until the sulci appear in the sucker. We continue downwards till the lateral part of the fourth ventricle is opened (Figs. 2 and 3). By this means the dentate nucleus is almost totally removed. It is very important that the borders of the cavity should be distinguished after 


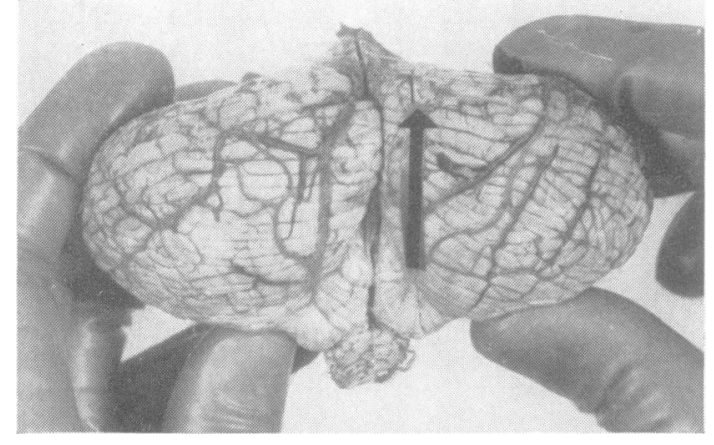

FIG. 1.-The arrow shows the line of incision.

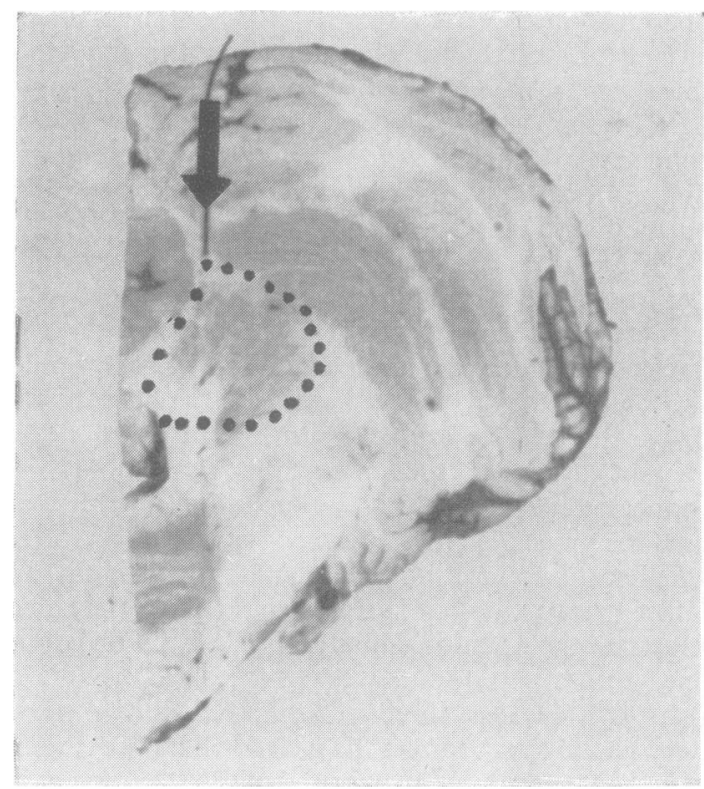

FIG. 2.-An arrow indicates the direction of penetration and a dotted line the part removed.

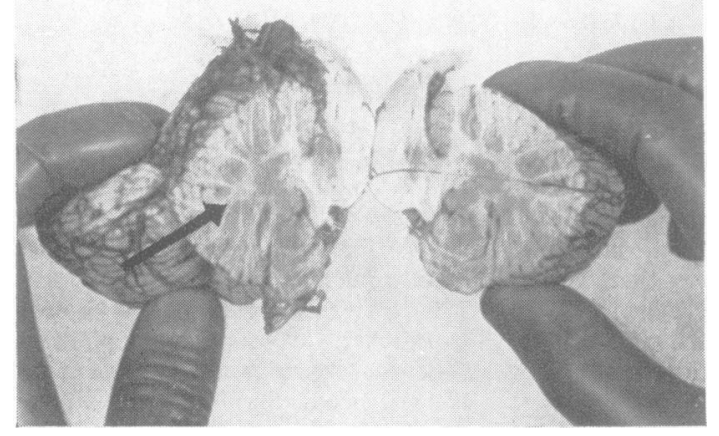

FIG. 3.-Sagittal section in the plane of the incision, an arrow indicating the direction of penetration. removal of the white matter, for only in one of the casese was the structure of the dentate nucleus discernible (the first of our three cases). After the operation the cerebrospinal fluid empties from the fourth ventricle and if the veins of the sulci are treated carefully, bleeding is minimal. Tremor is registered by means of an electromyograph before and after the operation. In the courseof the operation the dentate nucleus is stimulated at periods lasting 1-3 msec. at frequencies of 4 to 30 per. sec. and 3 to 6 volts in amplitude (square impulse) and the changes of tremor activity in the upper extremity are? registered.

\section{Case Reports}

Case 1.-A woman aged 56 years had had "Spanish flu" with high fever in 1930 . She recovered withouth complaints or symptoms. Fifteen years later the extre $\mathcal{S}$ mities began to shake, posture became rigid, speech $\overrightarrow{0}$ drawling, and gait slow. She had difficulties when eatingand hypersalivation. Between 1950 and 1956 she was $\vec{\omega}$ treated several times in neurological institutes, but only? transitory improvement was achieved. The symptoms? were more marked on the left side. On admission tơ our institute hypaesthesia of the left face and a reduced corneal reflex were observed on the left side. The leftiv side of the body exhibited reduced sensation to pain and touch. There was considerable muscular rigiditycu all over the body and cog-wheel symptoms in theo extremities. Bilateral tremor at rest was more marked. on the left side. Gait was composed of small steps. $Z$ Her speech was slurred and difficult to understani She had a mask-like facies. Also her face was coverd with a greasy secretion. There were reports of occasiogite oculogyral spasms. Electroencephalograms, radiograp的.of the skull and cerebrospinal fluid, ophthalmological, $\square$ and otoneurological examinations did not show any remarkable abnormality. Slight diffuse dilatation of the ventricular system was found on lumbar pneumo-o, encephalography.

Electromyograms taken before the operation registered the tremor on both sides, especially on the left where it $\vec{\overrightarrow{ }}$ was present at rest as well as during voluntary activity. The frequency of the tremor was 7-8 per sec., and its amplitude showed a marked increase during voluntary innervation, but the activity of the innervated muscle did:not surpass that of other muscles and the tremor showed $\overline{ }$ even at such times a marked antagonistic distribution.

As rigidity and hypokinesis were dominant among the symptoms, it seemed to be a case of the extrapyramidal syndrome suitable for approaching surgically by way of the cerebellum.

On January 15, 1958, the left dentate nucleus was removed. After the operation hypotonia on both extremities of the left side was detected; a few days $D$ later it increased. The patient exhibited no other cerebellar symptoms. The tremor on one side became less $N$ and the gradual improvement continued. Hypokinesis. remained. The patient could perform fine movements $N$ more easily, but her skill at doing them did not show anyN appreciable change. Though the patient registered the improvement, she continued to complain about symptoms of hypokinetic origin. 
According to the electromyogram taken after operation on February 7 the amplitude of tremor decreased approximately to half; the frequency did not change. During voluntary innervation tremor activity was less marked and the activity of the primary innervated muscle dominated. Almost two and a half years after the operation a control electromyogram was taken which showed further diminution of the tremor at rest to a tenth of its pre-operative activity. Frequency was 6 per sec. During voluntary innervation the activity of the primary innervated muscle was conspicuous. Clinical examination showed no rigidity. The patient's complaints and symptoms were chiefly derived from hypokinesis which could be controlled by drugs to some extent.

Examinations carried out after operation showed steady improvement regarding the rigidity and tremor, but for hypokinesis it showed periodical changes and thereby hindered the patient's movements.

Case 2.-S.M., a woman, aged 33 years, at the age of 6 had had a chronic disease with fever and disturbance of articulation and transitory abasia. At the age of 31, tremor of the fingers of the right hand began; pains in the right upper extremity and gradual weakening of the right extremities occurred. Tremor of the extremities increased to such a degree that the patient was unable to continue her work. In the course of the following years she was several times in hospital and received the most variable treatments, but no essential change occurred. On admission to our institute there was slight paresis of the right facial muscle and the tendon reflexes of the right side were enhanced, especially in the right hand. Moderate tremor at rest of the right upper extremity was observed, and from time to time rapidly increased in both extremities. Tremor fits occurred with variable frequency, but always as a result of excitement. Muscular hypertonia was not detected; the patient was unable to perform fine movements on the right side. Gait was rigid and spastic, though muscular hypertonia of the lower extremities was not observed. Imbecility was reported. The laboratory, radiological, ophthalmological, and otoneurological examinations showed no impairment of other systems. The patient, presumably as a consequence of an encephalitis hemisyndrome, had developed tremor at rest which was aggravated by action. As she showed no signs of improvement with drug treatment and was unable to work, we decided to perform stereotactic pallidothalamotomy. As repeated lumbar and cisternal pneumography failed to outline the ventricles, we dropped the idea of stereotactic operation, and the good results obtained by previously performed cerebellar operation encouraged us to remove the dentate nucleus of the affected side. Accordingly, on October 2, 1958, the dentate nucleus of the right side was removed. After operation the disturbance of movement completely ceased. Marked hypotonia of the right extremities was observed. Two or three days later intention uncertainty of the right upper extremity appeared and hypometria and ataxia of speech were found. Two weeks later intention uncertainty increased to intention tremor; later on tremor occurred also at rest. When the patient was made to leave her bed the disturbances of gait found before the operation disappeared. At first, on account of a strain to the right, the patient could walk only with support; a few days later the strain disappeared and her gait became perfectly undisturbed. Tremor of the right upper extremity remained unchanged or rather seemed somewhat increased. In January, 1959, the patient was sent for physiotherapy. Her tremor could be influenced to a certain extent by Artane. No tremor fits occurred after the operation. According to the electromyograms obtained before the operation, tremor at rest was about 300 microvolts in amplitude and 7 frequencies per sec. After the operation the amplitude of tremor was about 1,000 microvolts and the frequencies decreased to 5 per sec. The other symptoms, hypometria and ataxia of speech, improved.

On admission to our institute on June 18, 1960, the tendon reflexes were equal on both sides, tremor invariably more marked than before the operation, but the state of movement remained unchanged. Ataxia of speech had improved considerably, but was still perceptible; no disturbance of gait was recorded. Fits of tremor no longer occurred. With voluntary movement the tremor was distinctly diminished.

Case 3.-Dr. H.S., a man aged 52, could not give reliable information concerning former illnesses. The symptoms first began in 1951, when he noticed a slight tremor in the left upper extremity; as it increased, tremor occurred also in the left lower limb. Between 1954 and 1957 tremor extended to the right extremities. The patient's limbs became rigid as the tremor proceeded; he could hardly move and could not remain in a lying position for long because unbearable pain spread all over his body. On admission marked tremulousness of the tongue and lips was observed. There was tremor at rest of all extremities, more marked on the left side. The Achilles reflex on the left side was missing; otherwise no impairment of the reflexes could be detected. Muscular rigidity was universal, but was more severe on the left side. The patient could perform rough movements, but was unable to carry out fine movements. The attitude of the hands was of " main d'accoucheur". His posture was flexed and his gait rigid, composed of small steps. He had a mask-like face and his speech was quiet and slow. Rigidity diminished slightly after treatment with Artane, but responded in no other respect to any kind of drug treatment.

On account of a severe Parkinsonian syndrome, more marked on the left, and considering the fact that there was hardly any response to drug treatment, we decided to perform a stereotactic operation on the right side. On May 8, 1959, an electrolytic pallidothalamotomy was made on the right side (ansa lenticularis, the internal part of the pallidum in the ventrolateral nucleus of the thalamus). After pallidotomy rigidity decreased on the left; after thalomotomy tremor fits occurred and decreased perceptibly only a few days after the operation. Speech improved and the patient could better perform fine movements on the left side. About three months later gait improved, then gradually his condition worsened. Rigidity reappeared and became more marked on the right side. Electromyograms also proved the increase of 
tremor, and clinical examinations, increased rigidity. In view of the fact that the effect of thalamopallidotomy was only temporary, and, in addition to the tremor, rigidity had also become prominent, we dropped the idea of a new sterotactic operation, but decided to remove the dentate nucleus of the right dise, where rigidity and tremor proved more severe. The operation was performed on March 17, 1960. During the operation the dentate nucleus was stimulated (3-6 v. amplitude, frequency $6 \mathrm{msec}$, periods $3 \mathrm{msec}$. square impulses), after two to three impulses tremor decreased on the right side, then it stopped. Using $3 \mathrm{v}$. this process was less definite, but still existed. After the operation marked hypotonia was observed in the right upper and lower extremities; the tremor ceased completely. Voluntary movements could be well performed but the "main $d$ ' accoucheur" attitude of the hands and hypokinesia remained, in spite of the fact that fine voluntary movements, if only to a small degree, could be performed. In a week or so, hypertonia appeared occasionally in some muscles, but this remained transient and ceased after a few passive movements. About a week later slow undulating movements could be observed in the right extremities at rest. According to the control electromyograms the occasional movements corresponded to 3 per sec. tremor activity. The frequency of tremor before the operation was 6 per sec. There was a marked difference between the amplitude of tremor activity before and after operation. After the operation tremor activity during voluntary movement became unstable and for most of the time was not distinguishable in the normal interference pattern.

Control examinations carried out on June 17, 1960, showed hypotonia of the right upper and lower limbs invariably and especially marked in comparison with the left extremities. There were long tremor-free periods of the extremities, especially in the upper limb, followed by periods of tremor. Nevertheless, during these periods tremor was also less marked on the right side than on the left. The difference of frequencies remained unchanged.

\section{Discussion}

Hypotonia is a fundamental constituent of the neocerebellar syndrome. It is most marked in the human and progressively less noticeable as we proceed backwards in phylogenesis. It was the most prominent symptom after operation in our patients, appearing in each of our cases. It was permanent. It was more marked than after our pallido-thalamic operations, especially in our third patient, whose rigidity could with difficulty be overcome by passive movement. Improvement set in immediately after the operation and not only was the normal tone achieved but hypotonia developed. Therefore it appears that the tone-regulating function of the neocerebellum has the same tendency in Parkinsonian disease as in the intact system. At the same time hypotonia must be considered as a real deficiency symptom of the cerebellum, because it appears immediately after the operation, it remains after the shock period, and it remains unchanged at the beginning of the period of compensation and late shows only gradually a slight decrease.

Sherrington (1898) proved that stimulation of the anterior cerebellar lobe decreases decerebrate rigidity and its removal increases it. Similar resultis were obtained with anaemic decerebrate preparation of Pollock and Davis (1927), where ischaemia of the anterior cerebellar lobe increased further the decerebrate rigidity (Stella, 1944; Granit, Holmgrens and Merton, 1955). These cases, however, do not contradict each other because Parkinsonian rigidit is not the same as decerebrate rigidity (von Kiss an Fényes, 1934; Mettler, 1957). In our cases the intention was to eliminate the neocerebellum (nucleus dentatus), while in decerebrate experiment it is the anterior cerebellar lobe which is disconnected? It is important to note that after the removal of the dentate nucleus the spastic gait of our second patient ceased and this effect proved to be prolonged.

Regarding tremor, in two of our patients (Cases 1 and 3), especially in Case 3, there was a marketip diminution after removal of the dentate nucleuș This decrease became even more marked two and $\overline{\mid c}$ half years later in the case of our first patient. Ifip the second patient the amplitude of tremor increased considerably, but in both the second and the third case the frequency of tremor was markedly decreasects

Clinically, the tremors occur in two forms wifc are easy to distinguish from each other. In the case the tremor is more marked when the patient of at rest and during action it ceases or decreases. Im the second case, at rest, there is no tremor activit and it occurs and increases during action. The firs is the Parkinsonian and the second the cerebellay type of tremor. Undoubtedly, these two tremors aro distinguishable in their appearance, but electromyo graphic examinations do not separate the two types of tremor from each other. The distinction is made possible by other symptoms of the syndrome. This uncertainty exists not only in the distinction of clinical symptoms, but also regarding the site of origin and character of tremor connected with i. Mettler and his collaborators (1957) observed that after the removal of the nucleus dentatus or after the lesion of the brachium conjunctivum true Parkinsonian tremor (at rest) appears, and in their opinion Parkinsonian tremor is probably of this origin. Delmas-Marsalet and van Bogaert's un paralleled operations tend to justify this theory, for. on coagulating the nucleus dentatus they found thap the Parkinsonian tremor was increased.

Peterson, Magoun, McCulloch, and Lindsle N (1949) considered the brain-stem reticular substanco to be responsible for the occurrence of tremors, an Alexander, Székely, and Spiegel (1959) observed that 
stimulating the nucleus dentatus increased the tremor originating from the brain-stem lesions; from this it follows that the removal of the nucleus dentatus should diminish or abolish the tremor. Cooper's operations on the thalamus support the theory that the absence of cerebellar influence decreases the tremor. Wycis, Székely, and Spiegel (1957) in another series of experiments found that on cutting through the brachium conjunctivum tremor could be produced by stimulating the reticular substance of the brain-stem. It is evident that the occurrence of tremor is a combined phenomenon. It must depend on the failure of a system not quite understood which may cause tremor, while a lesion of this system may lead to the decrease of tremor. According to Walshe (1927) and Demole (1927), cerebellar tremor is the result of insufficient cortical cerebral compensation. According to Aring and Fulton (1936), only complete removal of the motor area can decrease cerebellar tremor while its partial removal rather increases it. On the basis of the findings of Bucy and Case (1937) and of Walker (1955) it is known that the injury of the motor cortex and of the corticospinal tract diminished Parkinsonian tremor, a diminution proportional to the grade of lesion of the motor cortex. In this respect our second case is of interest. This patient showed the most marked signs of a cortical lesion and after removal of the nucleus dentatus tremor markedly increased. After the operation the tremor was first of a cerebellar type but changed gradually to the typical Parkinsonian tremor. Experimental examinations show that the site of the lesion does not determine the character of the tremor. The above case shows that if tremor already exists, the lesion of another potentially tremor-causing site does not necessarily change its character.

It is interesting to note that in the case of our two patients, besides the marked hypotonia, there were no other cerebellar symptoms; in the third case, at the most there was slight hypometria. As for the second patient, the cerebellar symptoms began to develop only on the third and fourth day, suggesting that in the development of cerebellar symptoms the compensatory mechanism plays an important role. Inability to perform fine movements cannot be estimated as a cerebellar symptom, because such impairment existed also before the operation and there was if anything a slight improvement after the cerebellar operation.

Our cases confirm the opinion that the rigidity, hypokinesis, and tremor are not strictly connected with one another because when rigidity markedly decreases, hypokinesis does not show substantial improvement, and in two cases decrease of rigidity was not followed by increase but by decrease of the tremor.

\section{Summary}

The nucleus dentatus on one side was removed in three cases of the Parkinsonian syndrome. In all three cases a marked decrease of tone and considerable diminution of rigidity was observed. Tremor was markedly decreased in two cases and increased in one. When tremor and rigidity were diminished, the results were prolonged. Other Parkinsonian symptoms were not substantially influenced by surgical interventions on the cerebellum. After removal of the nucleus dentatus hypotonia is the only genuine cerebellar deficiency symptom which appears.

From a study of these cases it seems that rigidity, tremor, and hypokinesis are phenomena of comparatively independent existence varying independently of each other.

\section{REFERENCES}

Alexander, G. L., Székely, E. G., and Spiegel, E. A. (1959). Confin. neurol. (Basel), 19, 454.

Aring, C. D., and Fulton, J. F. (1936). Arch. Neurol. Psychiat. (Chicago), 35, 439.

Bravo, G. J., and Cooper, I. S. (1959). J. Neurol. Neurosurg. Psychiat., 22,1 .

Bucy, P. C., and Case, T. J. (1937). Arch. Neurol. Psychiat. (Chicago), 37, 983 .

Carrea, R. M. E., Reissig, M., and Mettler, F. A. (1947). J. comp. Neurol., 87, 321.

$\longrightarrow$, (1955). Ibid, 102, 151.

Delmas-Marsalet, P., and Bogaert, L. van (1935). Rev. neurol., 64, 728.

Demole, V. (1927). Schweiz. Arch. Neurol. Psychiat., 20, 271.

Fulton, J. F. (1949). Physiology of the Nervous System, 3rd ed. Oxford University Press, New York.

Granit, R., Holmgren, B., and Merton, P. A. (1955). J. Physiol. (Lond.), 130, 213.

Kiss, P. von, and Fényes, I. (1934). Mschr. Psychiat. Neurol., 90, 149.

Mettler, F. A. (1957). Premier Congrés international des Sciences Neurologiques. Bruxelles, 1957. Première Journée Commune: Rapports et Discussions, 1, 12. Les Editions Acta Med. Belgica, p. 11. Brussels.

Peterson, E. W., Magoun, H. W., McCulloch, W. S., and Lindsley, D. (1949). J. Neurophysiol., 12, 371.

Pollock, L. J., and Davis, L. (1927). Brain, 50, 277.

Sherrington, C. S. (1898). J. Physiol (Lond.), 22, 319.

Stella, Q. (1944). Atti Soc. med.-chir. Padova, 22, 5.

Stella, Q. (1944). Atti Soc. med.-chir. Pad

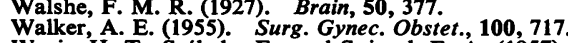

Wycis, H. T., Székely, E., and Spiegel, E. A. (1957). J. Neuropath. exp. Neurol.. 16, 79 . 